\title{
Cerâmicas odontológicas: Classificação, propriedades e indicações e protocolo de
}

\section{cimentação}

\author{
Dental ceramics: Classification, properties and indications and cementation protocol \\ Cerâmica dental: Clasificación, propiedades e indicaciones y protocolo de cementación
}

Recebido: 12/05/2021 | Revisado: 17/05/2021 | Aceito: 20/05/2021 | Publicado: 07/06/2021

\author{
Maria Rita Soares Gontijo Brandão \\ ORCID: https://orcid.org/0000-0002-8404-9762 \\ Faculdade Patos de Minas, Brasil \\ E-mail: mariarita.gsb10@hotmail.com \\ Taís Alves dos Reis \\ ORCID: https://orcid.org/0000-0003-3936-7312 \\ Faculdade Patos de Minas, Brasil \\ E-mail: taisareis@yahoo.com \\ Cláudia Maria de Oliveira Andrade \\ ORCID: https://orcid.org/0000-0003-4529-8106 \\ Faculdade Patos de Minas, Brasil \\ E-mail: claudia.andrade@faculdadepatosdeminas.edu.br \\ Elisabete Aparecida Rosário de Sousa \\ ORCID: https://orcid.org/0000-0003-3041-3415 \\ Faculdade Patos de Minas, Brasil \\ E-mail: elisabete.sousa@faculdadepatosdeminas.edu.br
}

\begin{abstract}
Resumo
As cerâmicas dentais são conhecidas pela sua excelência em reproduzir artificialmente os dentes naturais. Atualmente existem diversos tipos de cerâmicas e métodos de obtenção que entregam diferentes resultados em relação à resistência e a translucidez. Está variedade acaba gerando a necessidade de o profissional estar constantemente se aprimorando para que consiga indicar corretamente e trabalhar com os diversos materiais existentes no mercado. Neste trabalho, são apresentadas as classificações, propriedades, indicações e protocolos de cimentação, das cerâmicas feldspáticas convencionais, as infiltradas por vidro, as associadas a metais e as aluminizadas que foram projetadas objetivando uma resistência duas vezes maior que as feldspáticas convencionais. Este trabalho tem como objetivo a formulação de um agregado de informações acerca das diferentes cerâmicas dentais existentes, fornecendo assim um conjunto de informações que poderá auxiliar tanto acadêmicos de odontologia como os profissionais da área, a realizar a escolha do material ideal para cada situação. Foi realizada uma revisão de literatura narrativa qualitativa, utilizando-se como base bibliográfica artigos científicos disponíveis nos bancos de dados online PubMED, Lillacs, BVSsalud e Scielo, apresentados em português e inglês.
\end{abstract}

Palavras-chave: Cerâmica; Ligas metalo-cerâmicas; Coroas; Odontologia.

\begin{abstract}
Dental ceramics are known for their excellence in artificially reproducing natural teeth. Currently there are several types of ceramics and methods of obtaining that deliver different results in terms of strength and translucency. This variety ends up generating the need for the professional to be constantly improving so that he can correctly indicate and work with the various materials available on the market. In this work, the classifications, properties, indications and cementation protocols are presented, of conventional feldspathic ceramics, those infiltrated by glass, those associated with metals and aluminized ones that were designed aiming at a resistance twice greater than conventional feldspathics. This work aims to formulate an aggregate of information about the different existing dental ceramics, thus providing a set of information that can help both dentistry students and professionals in the field, to make the choice of the ideal material for each situation. A qualitative narrative literature review was carried out, using as bibliographic basis scientific articles available in the online databases PubMED, Lillacs, BVSsalud and Scielo, presented in Portuguese and English.
\end{abstract}

Keywords: Ceramics; Metal ceramic alloys; Crowns; Dentistry.

\section{Resumen}

Las cerámicas dentales son conocidas por su excelencia en la reproducción artificial de dientes naturales. Actualmente existen varios tipos de cerámicas y métodos de obtención que entregan diferentes resultados en cuanto a resistencia y translucidez. Esta variedad acaba generando la necesidad de que el profesional esté en constante mejora para poder 
indicar correctamente y trabajar con los distintos materiales disponibles en el mercado. En este trabajo se presentan las clasificaciones, propiedades, indicaciones y protocolos de cementación, de las cerámicas feldespáticas convencionales, las infiltradas por vidrio, las asociadas a metales y las aluminizadas que fueron diseñadas con una resistencia dos veces mayor que los feldespáticos convencionales. Este trabajo tiene como objetivo formular un agregado de información sobre las diferentes cerámicas dentales existentes, proporcionando así un conjunto de información que puede ayudar tanto a los estudiantes de odontología como a los profesionales en el campo, a realizar la elección del material idóneo para cada situación. Se realizó una revisión bibliográfica narrativa cualitativa, utilizando como base bibliográfica artículos científicos disponibles en las bases de datos en línea PubMED, Lillacs, BVSsalud y Scielo, presentados en portugués e inglés.

Palabras clave: Cerámica; Aleaciones de cerámica y metal; Coronas; Odontología.

\section{Introdução}

A nomenclatura cerâmica tem origem no grego, advindo originalmente da palavra Keramos a qual significa argila (Gomes, Assunção, Rocha, \& Santo, 2018). Existem indícios de aproximadamente 13 mil anos os quais demonstram que já se utilizava cerâmicas para diversos fins (Gomes et al, 2018; Namoratto, Ferreira, Lacerda, Sampaio Filho, \& Ritto, 2013). No século X os chineses já possuíam o domínio sobre a confecção de cerâmicas, as quais já apresentavam suas partes internas firmes e de coloração branca. A tecnologia para a confecção das cerâmicas chegou a Europa aproximadamente 7 séculos depois (Gomes et al, 2018). Apenas em 1720 os europeus conseguiram criar uma porcelana translucida e fina capazes de competir com as porcelanas chinesas compostas por feldspato, e oxido de cálcio submetidos a queima a altas temperaturas (Gomes et al, 2018).

Apenas 54 anos depois em 1774 um francês chamado Alexis Duchateau, descontente com suas próteses totais feitas com dentes de marfim, resolveu iniciar produção de novas prótese confeccionadas com cerâmica, material escolhido após análise de sua resistência ao manchamento e abrasão, além de sua durabilidade, aspectos já demonstrados quando empregadas em objetos domésticos (Gomes et al, 2018). Com a ajuda de Nicholas Dubois de Chemant, Alexis introduziu as cerâmicas na área odontológica (Gomes et al, 2018).

Próximo ao século XX surgiram as próteses parciais fixas confeccionadas em cerâmica. Estas receberam a nomenclatura de coroas de jaqueta e se difundiram rapidamente entre os profissionais da área odontológica (Aguiar, Rodrigues, Lopes, Silveira Junior, Soares, \& Novais, 2016; Gomes et al, 2018)

$\mathrm{Na}$ Inglaterra foram desenvolvidas as coroas feldspáticas, nas quais se incorporou entre 40 e $50 \%$ de cristais de alumina, com o intuito de adquirir uma maior resistência para as coroas de jaqueta (Gomes et al, 2018).

Simultaneamente a evolução das cerâmicas odontológicas, ocorreu o desenvolvimento dos agentes cimentantes, visando a longevidade das restaurações, retenção dos núcleos e das restaurações indiretas na cavidade oral (Aguiar et al, 2016; Amoroso, Ferrira, Torcato, Pellizzer, Mazaro, \& Gennari Filho, 2012).

Para se realizar uma adequada cimentação é necessário um bom tratamento, tanto das superfícies do substrato dental, quanto das superfícies da restauração, o qual pode variar de acordo com as características do sistema adesivo e das características particulares do agente cimentante (Aguiar et al, 2016; Amoroso et al, 2012, Pinho, 2015).

As cerâmicas odontológicas estão sendo cada vez mais requisitadas nos consultórios, principalmente devido à suas características que mimetizam as estruturas dentais, biocompatibilidade e características biomecânicas (Aguiar et al, 2016; Garcia, Consani, Cruz, \& Souza, 2011; Gomes et al, 2018; Monteiro, Oliveita, Steinhauser, \& Campos, 2017; Guarnieri et al, 2021; Pinho, 2015; Silva, 2019). Sua aplicação clínica consagrou-se por apresentar várias propriedades desejáveis, de forma semelhante aos dentes naturais, dentre as quais se destacam: translucidez, fluorescência, estabilidade química, coeficiente de expansão térmica linear próxima ao da estrutura dentária, compatibilidade biológica, assim como maior resistência à compressão e à abrasão (Aguiar et al, 2016; Garcia et al, 2011; Gomes et al, 2018; Monteiro et al, 2017; Pinho, 2015; Silva, 2019).

A busca atual, por restaurações estéticas tem resultado em um aumento no uso de cerâmicas dentais, antes restritas apenas a tratamentos em regiões anteriores, e hoje também abrangendo a área posterior (Aguiar et al, 2016; Amoroso et al, 2012; 
Gomes et al, 2018; Monteiro et al, 2017). Vários materiais cerâmicos e novas técnicas têm sido desenvolvidos durante as últimas décadas, uma vez que, as propriedades dos materiais cerâmicos tradicionais tinham limitada indicação para restaurações de maiores extensões devido as forças excessivas (Aguiar et al, 2016; Amoroso et al, 2012; Gomes et al, 2018; Monteiro et al, 2017).

Este trabalho objetiva a formulação de um agregado de informações acerca das diferentes cerâmicas dentais existentes, fornecendo assim um conjunto de informações que poderá auxiliar tanto acadêmicos de odontologia como os profissionais da área, a realizar a escolha do material ideal para cada situação.

Foi realizada uma revisão de literatura narrativa qualitativa, utilizando-se como base bibliográfica artigos científicos disponíveis nos bancos de dados online PubMED, Lillacs, BVSsalud e Scielo, apresentados em português e inglês.

\section{Metodologia}

Foi realizada uma revisão de literatura narrativa qualitativa, utilizando-se como base bibliográfica artigos científicos disponíveis nos bancos de dados online PubMED, Lillacs, BVSsalud e Scielo, apresentados em português e inglês (Estrela, 2018).

\section{Revisão da literatura}

A classificação das cerâmicas pode ser descrita de acordo com a sua composição, facilitando suas indicações clínicas que estão intimamente relacionadas com a composição, cor do substrato, área a ser restaurada e o tipo de restauração (coroa total, faceta, laminados cerâmicos) (Gomes et al, 2018; Monteiro et al, 2017). Os critérios que são utilizados para diferenciar materiais cerâmicos são baseados na fase ou fases presentes em sua composição química (Gomes et al, 2018; Monteiro et al, 2017). No que diz respeito às propriedades mecânicas, alguns parâmetros como força medida in vitro, resistência à compressão e abrasão, grau de translucidez, técnicas de fabricação também diferenciam as cerâmicas (Gomes et al, 2018; Monteiro et al, 2017).

\subsection{Cerâmicas com matriz de vidro}

A família das cerâmicas com matriz de vidro é subdividida em três subgrupos: cerâmica feldspática de ocorrência natural, cerâmica infiltrada por vidro e cerâmica aluminizadas (Silva, 2019).

As cerâmicas feldspaticas são cerâmicas compostas por uma base de vidro não metálica (Silva, 2019). Estás são constituídas por uma porção expressiva de feldspato natural que é formado por uma combinação de aluminossilicatos de sódio, potássio, quartzo e argila/caulim (Silva, 2019; Scherrer, Denry, \& Wiskott, 1998).

A translucidez da cerâmica é fornecida pelo quartzo, o qual representa entre 55 a $65 \%$ da matriz, porém este componente não confere uma adequada resistência a cerâmica e por isso, se utiliza a alumina como reforço, está representa entre 20 e $25 \%$ da base, já o caulim representa apenas $4 \%$ da mistura, uma vez que este apresenta propriedades opacas, as quais podem interferir no resultado estético translucido final, porém a este é atribuído a importante função de ligar as partículas da cerâmica que se encontram frouxamente ligadas (Silva, 2019). Estas cerâmicas são indicadas para restaurações metalocerâmicas e facetas laminadas. (Garcia et al, 2011; Hilgert, Junior, Vieira, Gernet, \& Edelhoff, 2008; Silva, 2019).

Estas cerâmicas possuem como vantagens a ausência da necessidade de equipamentos especiais, podem ser aplicadas em camadas finas e apresentam boa estética por oferecerem ótimas qualidade ópticas (Guazzato, Albakry, Ringer, \& Swain, 2004; Silva, 2019; Strassler \& Nathanson, 1989). São contraindicadas em situações de maior exigência funcional por possuírem baixa resistência a tração, altíssima friabilidade e desgaste dos dentes antagonistas (Guazzato et al, 2004; Silva, 2919; Strassler \& Nathanson, 1989). 
Os protocolos de cimentação das cerâmicas feldspáticas, demandam diferentes tempos de aplicação do ácido fluorídrico, dependendo do material utilizado (Hilgert et al, 2008; Rizkalla \& Jones, 2004; Sgrott et al, 2021). As cerâmicas feldspáticas convencionais são condicionadas utilizando ácido hidrofluoridrico a 9,5\% durante 2 minutos, já as reforçadas com leucita são expostas por 60 segundos e as que possuem dissilicato de lítio ficam em contato com o agente condicionante por apenas 20 segundos (Hilgert et al, 2008; Rizkalla \& Jones, 2004). Para se obter uma adequada resistência, as cerâmicas feldspaticas devem ser cimentadas sobre a estrutura dentaria remanescente, utilizando-se cimentos adesivos resinosos (Hilgert et al, 2008; Rizkalla \& Jones, 2004).

As cerâmicas infiltradas por vidro, foram introduzidas no mercado em 1995, apresentando o coping ou infraestrutura infiltrada por partículas de vidro (Hilgert et al, 2008; Rizkalla \& Jones, 2004). Este sistema possui três variações, de acordo com o seu principal componente de reforço, onde pode ser por alumina, indicado para confecção de coroas unitárias anteriores e próteses fixas de três elementos posteriores e anteriores até canino, reforçado por alumina e magnésio o qual apresenta maior translucidez e melhor aspecto estético sendo utilizado em regiões anteriores e reforçado por zircônia indicado para próteses de três elementos fixos na região posterior (Albakry, Guazzato, \& Swain, 2003; Garcia et al, 2011; Hornberger. Marquis, Christiansen, \& Strunk, 1996; Tian, Zhang, Zhang, \& Luo 1999). Estas possuem maior resistência que as feldspáticas, porém, perdem na translucidez. São indicadas para próteses de três elementos na região anterior e para confecção de núcleos cerâmicos. Tais cerâmicas podem ser cimentadas com cimento de fosfato de zinco (Rizkalla \& Jones, 2004).

Nas cerâmicas associadas a metais (zircônia), é incorporado uma maior quantidade de leucita em comparação as porcelanas feldspática para aumentar o coeficiente de expansão térmica (Gomes et al, 2018; Rosenblum \& Schulman, 1997). Estas possuem translucidez, são resistentes a compressão e apresentam baixa temperatura de fusão, porém, apresentam baixa resistência a flexão (Scherrer et al, 1998). Suas principais desvantagens são, falta de adesão a estrutura dentária remanescente, elevada solubilidade, possibilidade de provocar irritação pulpar e sensibilidade pós operatória devida ao baixo pH que apresentam (Gomes et al, 2018; Rosenblum \& Schulman, 1997). São indicadas como material para faceta das coroas metal-cerâmicas e próteses parciais fixas (Gomes et al, 2018). A cimentação final pode ser realizada com cimento de fosfato de zinco, devido ao seu baixo custo, facilidade de manipulação e boas características mecânicas (Namoratto et al, 2013).

As cerâmicas aluminizadas, foram desenvolvidas com o intuito de proporcionar o dobro de resistência a ruptura, uma vez comparadas as cerâmicas feldspáticas convencionais (Amoroso et al, 2012; Pinho, 2015). Encontra-se na sua composição, aproximadamente $50 \%$ de oxido de alumina com maior resistência a flexão (Amoroso et al, 2012; Pinho, 2015). No entanto, foi detectada uma perda de translucidez causada pela limitada transmissão de luz pelos cristais de alumina. Também foi observado que a resistência obtida ainda se demonstrava insuficiente para uso em regiões posteriores e para construção de próteses parciais fixas (Amoroso et al, 2012; Pinho, 2015). Estas por fim, acabaram possuindo indicação apenas para próteses de três elementos em região anterior e para a confecção de núcleos cerâmicos (Amoroso et al, 2012; Pinho, 2015). Estas cerâmicas podem ser cimentadas utilizando-se cimento de fosfato de zinco (Pinho, 2015).

Apesar dos cimentos convencionais como o fosfato de zinco ainda apresentarem resultados satisfatórios, estes vem sendo substituídos gradativamente pelos resinosos (Guazzato, Albakey, Ringer, \& Swain, 2004; Namoratto et al, 2013). Os cimentos resinosos se diferenciam dos cimentos convencionais devido a sua composição, se demonstrando semelhante as resinas compostas foto ativáveis, apresentando maior força de união e estética superior (Guazzato et al, 2004; Namoratto et al, 2013). O cimento resinoso de polimerização exclusiva pela luz é o indicado para cimentação de facetas cerâmicas, sendo estas restaurações relativamente finas e translúcidas; a luz atravessa completamente a restauração ocorrendo assim à completa conversão dos monômeros, conferindo a estabilidade de cor do cimento (Guazzato, 2004; Namoratto et al, 2013).

A força de união da cerâmica e do cimento, pode ser aumentada através de tratamentos químicos, como o 
condicionamento ácido da superfície cerâmica, obtenção de micro retenções, união mecânica através de processos físicos e pelo método triboquímico onde se aplica um jacto de partículas de óxido de alumínio recobertas com sílica (Namoratto et al, 2013).

\section{Discussão}

Para Gomes e colaboradores (2018), os materiais cerâmicos apresentam grandes propriedades mecânicas, as quais possibilitam, a confecção de restaurações cerâmicas livres de metal tanto para a região anterior quanto para região posterior. Estes autores enfatizam a necessidade de uma avaliação criteriosa para a seleção do sistema cerâmico adequado, levando em consideração não apenas a resistência mecânica do material, mas também a região em que este será empregado, além da forma de união que será realizada entre a estrutura dentária e a restauração indireta, garantindo desta forma a longevidade do tratamento.

Alguns autores (Namoratto et al, 2013; Pinho, 2015) atestam que devido aos diversos sistemas cerâmicos existentes atualmente no mercado, é de extrema importância que os profissionais da área odontológica, realizem constantes reciclagens a respeito de suas propriedades e indicações, para que sejam empregados em suas condições ideais aumentando desta forma a longevidade do tratamento, uma vez que esta durabilidade não depende exclusivamente do tipo de material empregado, mas também de sua aplicação em condições em que é adequado e do conjunto de habilidades do profissional operador.

Namoratto e colaboradores (2013), destacam que apesar dos cimentos tradicionais, tais como fosfato de zinco e ionômero de vidro ainda serem amplamente utilizado para restaurações indiretas em regiões posteriores, os cimentos resinosos e auto adesivos trouxeram novas técnicas, que permitem resultados extremamente estéticos, o que faz com sejam usados na grande maioria das vezes quando se trabalha com restaurações indiretas em regiões anteriores, principalmente na arcada superior.

Garcia e colaboradores (2011), afirmam que apesar dos sistemas cerâmicos atuais proporcionarem restaurações com aparência extremamente próxima a dos dentes naturais, possuírem resistência adequada e promoverem uma adequada adaptação nas margens, é imprescindível que o profissional conheça as características dos materiais que está fazendo uso para que seja possível alcançar o sucesso clínico.

Neto e colaboradores (2020), deixam claro que selecionar um sistema cerâmico baseado exclusivamente na sua translucidez pode ocasionar uma indicação incorreta, o que leva ao insucesso do tratamento. Os autores também salientam a necessidade de seguir a ordem correta dos protocolos de cimentação, bem como um bom conhecimento e domínio dos sistemas de cimentos resinosos para se obter sucesso clínico.

\section{Conclusão}

As cerâmicas odontológicas passaram por uma série de grandes mudanças, desde seu início com as cerâmicas feldspáticas convencionais.

O desenvolvimento das cerâmicas infiltradas por vidro conferia resistência que as cerâmicas feldspáticas tradicionais, porém apresentavam uma estética inferior.

As cerâmicas associadas a metais apresentam translucidez e resistência a compressão, porém possuem uma menor defesa contra a flexão.

Cerâmicas aluminizadas apresentam resistência superior as feldspaticas convencionais. Entretanto, apresentam perda de translucidez e a resistência obtida não foi a esperada.

Apesar de atualmente existir uma boa variedade de opções cerâmicas e destas possuírem propriedades interessantes as quais agradam tanto os cirurgiões dentistas quanto os pacientes, ainda não há uma opção ideal, a qual cumpra todos os prérequisitos desejáveis. 
Recomenda-se a realização de novos estudos, para que seja possível realizar o desenvolvimento de novas cerâmicas as quais superem as já existentes nos quesitos estética, resistência, conforto e praticidade.

\section{Referências}

Aguiar, E. M. G., Rodrigues, R. B., Lopes, C. C. A., Silveira Júnior, C. D., Soares, C. J., \& Novais, V. R. (2016). Diferentes sistemas cerâmicos na reabilitação oral: relato de caso. ROBRAC. 25(72), 31-36.

Albakry, M., Guazzato, M., \& Swain, M. V. (2003). Fracture toughness and hardness evaluation of three pressable all ceramic. dental materials. J dent. 31(3), $181-188$.

Amoroso, A. P., Ferreira, M. B., Torcato, L. B., Pellizzer, E. P., Mazaro, J.V. Q., \& Gennari Filho, H. (2012). Rev Odontol. Araçatuba (Online). 33(2), 19-25.

Estrela, C. (2018). Metodologia Científica: Ciência, Ensino, Pesquisa. Editora Artes Médicas.

Garcia, L. F. R., Consani, S., Cruz, P. C., \& Souza, F. C. P. P. (2011). Análise crítica do histórico e desenvolvimento das cerâmicas odontológicas. RGO (Porto Alegre). 59, 67-73.

Gomes, E. A., Assunção, W. G., Rocha, E. P., \& Santos, P. H. (2018). Cerâmicas odontológicas: o estado atual. Rev Cer. 54(331), 319-325.

Guarnieri, F. D. F., et al. (2021). Conservative management for ceramic laminate veneers using digital workflow: case report with 18 month fallow-up. RSD. $10(4), 1-12$.

Guazzato, M., Albakry, M., Ringer, S. P., \& Swain, M. V. (2004). Strength, fracture toughness and microstructure of a selection of all-ceramic materials. Part II. Zirconia-based dental ceramics. Dent mater. 20(5), 449-456.

Guzzato, M., Albakey, M., Ringer, S. P., \& Swain, M. V. (2004). Strength, fracture toughness and microstructure of a selection of all ceramic materials, part I, oressable and alumina glass infilted ceramics. Dent mater. 20(5), 449-456.

Hilgert, L. A., Júnior, S. M., Vieira, L. C. C., Gernet, W., \& Edelhoff, D. (2008). A escolha do agente cimentante para restaurações cerâmicas. Clínica Int J Braz Dent. 5(2), 194-205.

Hornberger, H., Marquis, P. M., Christiansen, S., \& Strunk, H. P. (1996). Microstructure of a high strength alumina glass composite. J Mater Res. 11(4), 855858 .

Monteiro, Y., Oliveita, J. M. V., Steinhauser, H. C., \& Campos, A. L. O. (2017). p. 1-2. Porto Alegre, RS, Brasil.

Namoratto, L. R., Ferreira, R. S., Lacerda, R. A., Sampaio Filho, H. R., \& Ritto, F. P. (2013). Cimentação em cerâmicas: evolução dos procedimentos convencionais e adesivos. Rev bras odontol. 70(2), 142-147.

Neto, J. M. A. S., Futado, K. R. S., Baumberger, M. C. A., Duarte, I. K. F., Trujillo, A. M., Alves, E. V. R., et al. (2020). Cerâmicas odontológicas: uma revisão de literatura. Rev Elet Acer Saud. (40), 1-10.

Pinho, I. V. (2015). Protocolos de cimentação definitiva nas cerâmicas feldspáticas e cerâmicas reforçadas. Dissertação. Universidade Fernando pessoa, Porto, MTS, Portugal.

Rizkalla, A. S., \& Jones, D. W. (2004). Identation fracture toughness and dynamic elastic moduli for comercial feldspathic dental porcelain materials. Dent mater. 20(2), 198-206.

Rizkalla, A. S., \& Jones, D. W. (2004). Mechanical properties of comercial hight strenght ceramic core materials. Dent mater. 20(2), 207-12.

Rosenblum, M. A. R. C. A., \& Schulman, A. L. L. A. N. (1997). A review of all ceramic retorations. J am dent assoc. $128(3), 297-307$.

Scherrer, S. S., Denry, I. L., \& Wiskott, H. W. A. (1998). Compararison of three fracture toughness testing techniques using a dental glass and a dental ceramic. Dent mater. 14(4), 246-255.

Sgrott, N. E., et al. (2021). Evaluation of self-etching primer on bond strength in feldspathic ceramic. RSD. 10(5), 1-8.

Silva, F. M. V. D. (2019). Todas as cerâmicas são iguais? Monográfia. Universidade Federal de Minas Gerais, Belo Horizonte, MG, Brasil.

Strassler, H. E., \& Nathanson, D. (1989). Clinical evaluation of etched porcelain veneers over a period of 18 to 42 months. J esthet restor dente. 1(1), $21-28$.

Tian, J. M., Zhang, Y. L., Zhang, S. X., \& Luo, X. P. (1999). Mechanical Properties and Microstructure of Alumina-Glass Composites. J Am Cream Soc. 82(6), $1592-1594$ 